\title{
Structural and biochemical insights of new atypical FPG/NEI DNA - glycosylases
}

\author{
A.V. Yudkina ${ }^{1,2,3 *}$, D.O. Zharkov ${ }^{1,2}$, M. Garcia-Diaz ${ }^{3}$ \\ ${ }^{1}$ SB RAS Institute of Chemical Biology and Fundamental Medicine, Novosibirsk, Russia \\ ${ }^{2}$ Novosibirsk State University, Novosibirsk, Russia \\ ${ }^{3}$ Stony Brook University, Stony Brook, US \\ *e-mail: yudkinaanya@gmail.com
}

Key words: DNA damage, DNA repair, formamidopyrimidine-DNA glycosylase, endonuclease VIII, protein crystalolography, Bacteroides thetaiotaomicron

Motivation and Aim: DNA glycosylases are enzymes that maintain genome integrity and they are a key element of base excision DNA repair. Due to their ability to recognize base lesions created by ionizing radiation, alkylating and oxidizing agents, these enzymes are still a subject of particular interest and can find use in different areas. Recently, by wholegenome sequencing of some bacteria reveal new Fpg/Nei-like DNA - glycosylases. However, these new Fpg/Nei-like enzymes show very low percentage of sequence identity to existing representatives of Fpg/Nei superfamily. In this work we aim to investigate structural and biochemical aspects of new Fpg/Nei-like DNA - glycosylases of different bacteria.

Methods and Algorithms: We have used molecular cloning and optimized protocol of protein purification to get new Fpg/Nei-like proteins. Using biochemical approaches, we have got first understanding of affinity and substrate specificity of these enzymes. By protein crystallography method we have crystallize and resolve the structures of new Fpg/Nei-like proteins from Bacteroides thetaiotaomicron.

Results: In the molecule of Fpg/Nei superfamily belonging proteins there are some structural features that provide the mechanism of catalysis. Most Fpg/Nei glycosylases have high-conservative PELPEVET $N$-terminal motif and use their $N$-terminal proline residue as a key catalytic nucleophile. Additionally, Fpg/Nei proteins have helix-twoturn-helix domain and zinc-finger motif to contribute residue to the active site. However, new Fpg/Nei-like proteins have atypical N-terminal residues such as isoleucine and lysine and significant diversity of $N$-terminal motif. We have crystallized Fpg/Nei-like protein from Bacteroides thetaiotaomicron with 2.0 A resolution and reveal the pretense of $\mathrm{Zn}$-finger domain in $\mathrm{C}$-terminal of protein, a-helix rich region in $\mathrm{N}$-terminal and some another Fpg-like features. However, substrate preferences make these proteins similar to Nei. Conclusion: We have revealed structural and biochemical aspects of new atypical Fpg/Nei-like proteins to determine relationships these proteins to known glycosylases families by protein crystallographic approach.

Acknowledgements: This work was supported by Russian Science Foundation (No. 14-24-00093). The authors thank Dr. M. Saparbaev for plasmids containing new Fpg-like proteins. The corresponding author thanks Fulbright Scholarship for opportunity to proceed experiments in the US. 